\title{
Médiévales
}

Langues, Textes, Histoire

54 | printemps 2008

Frères et sœurs

\section{Relations professionnelles et relations fraternelles d'après le journal de Lucas Rem, marchand d'Augsbourg (1481-1542)}

Professional and fraternal relations according to Lucas Rem's journal, merchant of Augsburg (1481-1542)

\section{Aude-Marie Certin}

\section{OpenEdition}

Journals

Édition électronique

URL : https://journals.openedition.org/medievales/4952

DOI : 10.4000/medievales.4952

ISSN : $1777-5892$

\section{Éditeur}

Presses universitaires de Vincennes

\section{Édition imprimée}

Date de publication : 1 juin 2008

Pagination : 83-98

ISBN : 978-2-84292-217-7

ISSN : 0751-2708

\section{Référence électronique}

Aude-Marie Certin, «Relations professionnelles et relations fraternelles d'après le journal de Lucas Rem, marchand d'Augsbourg (1481-1542) », Médiévales [En ligne], 54 I printemps 2008, mis en ligne le 10 septembre 2010, consulté le 22 avril 2022. URL : http://journals.openedition.org/medievales/4952 ; DOI : https://doi.org/10.4000/medievales.4952

Ce document a été généré automatiquement le 22 avril 2022

Tous droits réservés 


\title{
Relations professionnelles et relations fraternelles d'après le journal de Lucas Rem, marchand d'Augsbourg (1481-1542)
}

\author{
Professional and fraternal relations according to Lucas Rem's journal, merchant \\ of Augsburg (1481-1542)
}

Aude-Marie Certin

Les écrits privés (autobiographie, journaux ou livres de famille) sont une source inestimable pour étudier les relations entre frères et soeurs à la fin du Moyen Âge. Audelà des discours normatifs sur la famille, véhiculés notamment par les clercs, cette documentation permet d'aborder de façon plus fine et complexe les réalités familiales. S'y révèlent certains aspects de la vie quotidienne des familles, les relations entre leurs membres en fonction de leur âge et de leur sexe et l'évolution de leurs sentiments au fil du temps ${ }^{1}$. Si de tels écrits sont rares en France aux XIV et $\mathrm{Xv}^{e}$ siècles ${ }^{2}$, ils sont en revanche nombreux en Italie et en Allemagne à la même époque. Les sources italiennes nous sont aujourd'hui bien connues, principalement grâce aux travaux de Christiane Klapisch-Zuber sur les ricordanze italiens ${ }^{3}$. Ceci n'est en revanche pas le cas des sources allemandes du même type qui restent globalement méconnues en France, ce que l'on ne peut que grandement déplorer compte tenu de leur diversité et de leur richesse. Les écrits privés de laïcs aujourd'hui conservés en Allemagne se comptent en effet par dizaines et se présentent sous des formes multiples : Hausbücher (livre de maison), livres de famille, Geschlechtbücher (livre de lignage), autobiographies ou journaux ${ }^{4}$. Ces «témoignages de soi » peuvent aussi se trouver dans des sources non directement autobiographiques, de formes et de contenus très divers, comme des chroniques urbaines, des livres de compte, des lettres, des récits de voyage ou de pèlerinage ou des comptes-rendus de procès. Depuis les années 1990, ces sources ont été l'objet d'un fort regain d'intérêt dans l'aire germanique ${ }^{5}$ : un certain nombre de colloques et d'ouvrages 
collectifs ont été consacrés à ces "témoignages de $\operatorname{soi}^{6}$ » et plusieurs historiens ont récemment étudié certaines de ces sources dans l'optique d'une histoire de la famille ${ }^{7}$.

Ces écrits privés allemands émanent surtout des élites urbaines. Leurs auteurs habitent le plus souvent dans les grandes villes de l'Empire, comme Nuremberg, Francfort ou Augsbourg. Et la plupart appartiennent aux couches dominantes de la ville; ainsi des familles Stromer ${ }^{8}$, Rohrbach ${ }^{9}$, Tucher ${ }^{10}$ ou Fürer, véritables pépinières de chroniqueurs, où les membres du Conseil et les bourgmestres se comptent en grand nombre; ainsi également de Lucas Rem ${ }^{11}$ ou de Burkard Zink ${ }^{12}$, riches marchands d'Augsbourg ${ }^{13}$. Le plus souvent ce sont des hommes riches et puissants mêlant activités politiques et commerciales qui fixent par écrit leur histoire ou celle de leur famille pour leurs descendants ou pour leurs semblables ${ }^{14}$. Des profils différents de rédacteurs existent néanmoins, comme Albrecht Dürer, le célèbre peintre de la Renaissance, auteur d'une chronique familiale ${ }^{15}$ reprenant celle de son père.

3 Dans l'Allemagne de la fin du Moyen Âge, les enjeux de ces écrits privés sont multiples. En premier lieu, la chronique ou le livre de famille pourrait être comparé à l'acte testamentaire à une époque où le souci du salut est primordial. Il s'agit de bien agir de son vivant pour bien mourir, en l'occurrence assurer la mémoire de tous les événements importants de la famille et véhiculer en bon chrétien des modèles de comportement. Il s'agit aussi, en particulier pour les élites urbaines, d'affirmer l'identité d'un groupe familial et d'assurer les bases de son devenir ${ }^{16}$. Vecteur de la mémoire familiale, le livre de famille reprend les éléments qui en font la spécificité et la valeur: l'écrit rassemble en un espace unique tout le patrimoine de la famille. Fréquemment ces écrits sont aussi aménagés pour servir de preuves dans des démarches juridiques ou politiques potentielles. Il apparaît prudent de garder en mémoire les traces de ses origines, des héritages, mariages ou transactions, pour éviter les problèmes juridiques et assurer l'avenir de sa famille dans la sphère sociale et politique ${ }^{17}$. Cette primauté donnée au collectif familial fait de cette parole une mémoire utile, tant dans ses ambitions que par son contenu ${ }^{18}$. Un certain nombre de ces écrits, en particulier les "livres de famille " (Familienbücher) et les "livres de lignage" (Geschlechtbücher) s'inscrivent aussi dans le mouvement d'affirmation et de fermeture des élites urbaines. De véritables stratégies de transmission et de conservation de documents écrits se mettent en place ${ }^{19}$. L'écriture de ces livres de famille ou de lignage vise à prouver ou à forger de nobles et prestigieuses origines, seules à mêmes de légitimer l'appartenance au cercle de plus en plus étroit des élites. Enfin, l'ambivalence du rapport au marchand dans la société de la fin du Moyen Âge peut constituer une autre clé de lecture. Si le pouvoir social des marchands ne fait plus de doutes dans les faits, leur légitimité idéologique demeure problématique. Il leur est de ce fait préférable de laisser à la postérité le souvenir de leur éthique ou de l'exemplarité de leur comportement de chef de famille.

4 Prendre sa plume pour fixer des notes familiales ou autobiographiques s'inscrit souvent dans une continuité. C'est le cas par exemple pour le patricien de Francfort Bernhard Rohrbach ${ }^{20}$ qui dit rassembler les notes de son grand-père et de son père. Il en est de même pour le marchand d'Augsbourg Lucas Rem dont les premières notes sont la compilation d'une mémoire familiale écrite et orale. Le marchand reprend lui aussi les écrits de son grand-père ${ }^{21}$ puis évoque ce qu'il a appris de la bouche de son père ${ }^{22}$. Bernhard Rohrbach, Albrecht Dürer et Lucas Rem suivent en définitive « un principe médiéval d'appropriation de la mémoire antérieure ${ }^{23} »$. Écrire et fixer la mémoire 
relève de l'imitation de leur père ou de leur grand-père: c'est en soi une forme d'héritage entre père et fils qui perdure. Mais plus encore, c'est un devoir incombant au chef de famille qui se transmet en ligne masculine, du père à son fils devenu majeur ou père lui-même. Ainsi ces écrits manifestent et construisent tout à la fois une culture proprement masculine, nourrissant la hiérarchie médiévale entre homme et femme, père et mère dans la famille, mais également une hiérarchie entre les frères différenciant d'un côté l'aîné porteur de la mémoire familiale, de l'autre les cadets.

5 Aussi l'autorité acquise dans la fratrie par cet acte d'écriture est-elle immense. Si c'est l'âné qui la plupart du temps hérite des papiers de la famille, ce n'est pas toujours le cas : ainsi, chez les Rohrbach de Francfort, c'est Bernhard, plus jeune que son frère Heinrich de quatorze ans, qui entreprend la rédaction de la chronique familiale en reprenant les écrits de ses aïeux. Par ce geste, il se fait le véritable héritier de la tradition des pères du lignage et acquiert une autorité considérable dans sa famille, notamment dans la fratrie. Ceci est particulièrement patent quand il exclut son frère des cérémonies religieuses familiales jugeant sa piété trop indigne. L'organisation générale de l'ensemble de sa chronique va également dans ce sens; après l'évocation des pères de la famille sur quatre générations, ainsi que celle de son frère, Bernhard Rohrbach finit sur sa propre paternité, apparaissant comme le fidèle successeur des pères du passé. Chez les Rohrbach, le fait d'écrire l'histoire de la famille amène à devenir symboliquement le père de celle-ci et fait acquérir l'autorité sur tous les membres qui la composent, les frères entre autres. La mémoire cristallise les rivalités entre frères et la question de l'autorité dans la famille. Dans la famille de Lucas Rem, le travail d'écriture est pris en charge par l'aîné de la fratrie de façon traditionnelle, mais les enjeux de pouvoir y sont comparables. Nous verrons plus loin combien les hiérarchies au sein de la famille, et plus spécialement au sein de la fratrie, sont également au cœur des notes personnelles du marchand.

6 Le statut de chef de famille acquis symboliquement par l'écriture des écrits privés donne un poids très important au discours des rédacteurs. Il est un vecteur d'idéal social et familial, tant dans la sphère de la parenté, de la famille étroite que de la fratrie. Adressés à la postérité familiale et sociale, ces textes véhiculent les valeurs et les modèles de comportement qu'en bon chef de famille, le rédacteur juge devoir transmettre en fonction de son groupe social et de ses intérêts propres. Aussi ces écrits privés révèlent-ils les idéaux d'individus ou de groupes sociaux relatifs aux relations entre frères et soeurs, notamment en ce qui concerne les cadets et l'âné.

7 Dans ce vaste corpus de sources, les notes personnelles de Lucas Rem (1481-1542), marchand d'Augsbourg, sont particulièrement riches pour étudier les relations entre frères et sœurs. L'une des raisons majeures est que trois des frères Rem partagent une même activité professionnelle : le commerce. Les parcours de ces frères se suivent dans leurs années de formation au sein de la compagnie des Welser et ils se rapprochent encore plus à l'âge adulte, quand ils décident de fonder ensemble une compagnie de commerce. De ce fait la fratrie y est très souvent évoquée et le discours du marchand permet d'aborder les relations entre frères dans leurs aspects quotidiens et de réfléchir aux enjeux de la fraternité dans la famille et dans le monde professionnel ${ }^{24}$, en particulier en termes de hiérarchie et de pouvoir ${ }^{25}$. Ce « journal $»^{26}$ nous permettra de voir combien la sphère marchande peut être structurée par les liens fraternels. Le discours du marchand nous amènera également à nous demander dans quelle mesure les relations professionnelles se structurent par les relations familiales. Les hiérarchies 
au sein de la société de commerce se basent-elles sur les hiérarchies de la famille étroite, spécialement celle entre l'aîné et ses cadets? Peut-on envisager une sorte de confusion entre sphère familiale et sphère marchande, où la société de commerce prendrait des allures de famille étroite dominée par le frère aîné devenu chef de famille ? Le frère aîné devient-il une sorte de père dans sa société brouillant les places de chacun dans la famille ainsi que les différences entre frères, fils et associés, obligeant à redéfinir la notion de société (Gesellschaft)?

Le « journal » de Lucas Rem

Grand facteur de la société Welser à Venise, Lyon, Lisbonne et Anvers, puis fondateur, avec ses frères, de sa propre compagnie, Lucas Rem met par écrit des notes personnelles pendant environ quarante ans. Aucune information ne permet de déterminer avec certitude la date à laquelle il a commencé cette entreprise. Néanmoins le caractère plus détaillé de ses notes à partir de 1498 permet d'émettre l'hypothèse que le marchand a commencé son journal à cette date. Cette année-là, le jeune homme a dix-sept ans et intègre la puissante compagnie marchande des Welser où il commence sa carrière d'homme d'affaires. Concernant la fin de la rédaction de ce journal, les dernières notes écrites de sa main datent de septembre $1540:$ l'homme est alors âgé de cinquante-neuf ans et il meurt deux ans plus tard. Précisons que dans sa version dactylographiée $e^{27}$, ces notes personnelles comptent quatre-vingt pages.

Écrites en moyen haut-allemand, ces notes s'organisent en dix parties thématiques définies par lui : les origines de son lignage; sa propre vie (sa naissance, sa vie, ses voyages) ; des renseignements sur ses capitaux commerciaux, ses gains et ses dépenses ; un bilan des dépenses liées à son mariage; ses cadeaux de mariage; une liste de ses rentes et de ses biens mobiliers; des informations sur ses enfants non nés de son mariage (partie non conservée intégralement); des informations sur ses six enfants légitimes; la liste de ses employés; le compte de ses impôts à Augsbourg entre 1516 et 1534. Notons que c'est dans un style très comptable que cet homme évoque ce qui constitue à ses yeux les éléments les plus importants de son existence.

10 Lucas Rem, troisième du nom, est originaire d'une des familles les plus riches et renommées d'Augsbourg, dont l'activité principale est le commerce au long cours. Cette famille est liée aux autres grandes familles d'Augsbourg tant par des liens de commerce que par des liens d'alliance. La mère de notre chroniqueur est ainsi la fille de Lucas Welser et Ursula Lauginger, les Welser et les Lauginger étant deux grandes familles d'Augsbourg. Dès le $\mathrm{xv}^{\mathrm{e}}$ siècle, les Rem accumulent une belle fortune grâce à leurs activités de commerce et commencent à acquérir des terres et des seigneuries en dehors de la ville d'Augsbourg.

11 La fratrie à laquelle appartient Lucas Rem compte quatre garçons et une fille et l'on peut supposer que tous sont nés d'un même lit. Le prénom Lucas porté par l'auteur nous amène à supposer qu'il est aîné de cette fratrie. Leur père s'appelle en effet Lucas Rem or, selon les règles anthroponymiques de l'époque, c'est le fils aîné qui hérite du prénom $d u$ père. Les autres frères se nomment Gilg, Endris et Hans et la sœur, Magdalena. Cet ordre d'apparition nous incite à penser que c'est là l'ordre de naissance dans la fratrie, du moins pour les garçons ${ }^{28}$. Nous savons par ailleurs qu'Hans meurt en 1527, Endris en 1537. Ces deux frères, dont Lucas Rem semble le plus proche, sont marchands comme lui. Ils commencent leur carrière à l'instar de leur aîné comme facteurs de la compagnie des Welser, famille dont est issue leur mère. Libérés quelques années plus tard de leurs obligations à l'égard de cette société, ils créent avec Lucas 
leur propre compagnie, rejoints par deux associés extérieurs à leur famille. Gilg quant à lui est prêtre, puis il devient évêque de Chiemsee dès 1509 et enfin évêque de Salzbourg dans les années 1520. Sa sœur Magdalena est au couvent à Dillingen. Il n'est pas improbable que les carrières ecclésiastiques de Gilg et Magdalena relèvent de stratégies familiales visant à éviter autant que possible la division du patrimoine familial.

Dans le discours du marchand, les frères, notamment Hans et Endris, apparaissent comme un cercle privilégié d'affection et de solidarité. Si une précédente étude ${ }^{29} \mathrm{a}$ montré que la parenté éloignée ainsi que les parents de l'auteur ne sont que peu évoqués dans les notes du marchand, la fratrie y tient en revanche une place de choix. Alors que l'auteur n'évoque ses propres parents que dans $1 \%$ de son texte, on compte non moins de 70 occurrences relatives à ses frères et sœur dans ses notes, et sa fratrie est évoquée dans environ $10 \%$ du texte. C'est, à titre de comparaison, autant que les propres enfants de Lucas Rem, signe de l'importance de cette fratrie dans la vie de l'auteur. Les frères constituent ainsi pour Lucas Rem le second cercle de parenté après celui constitué par lui, sa femme et leurs enfants.

Si importante soit la place des frères et sœurs dans la vie quotidienne du rédacteur, il convient néanmoins de constater que le discours porté sur eux n'est en rien comparable à celui tenu sur les enfants de l'auteur. Tandis que Lucas Rem brosse un portrait physique et moral de chacun de ses enfants et dépeint les relations, parfois difficiles d'ailleurs, qu'il entretient avec chacun d'eux, il ne donne en revanche aucune information sur les caractères de ses frères, ni de détails sur les relations qu'il entretient avec eux. Les frères ne sont par ailleurs jamais évoqués dans leur vie privée, ni dans leur vie de famille, même s'ils ont femme et enfants comme nous le découvrons au moment des problèmes d'héritage. Lucas Rem n'évoque ses frères que dans la relation exclusive qu'il entretient avec eux, relation mêlant liens fraternels et liens professionnels. Ses frères ne semblent pas avoir de vie propre en dehors de leurs activités professionnelles et de leur fratrie; c'est en tout cas l'image que Lucas Rem tend à donner d'eux.

La fratrie, un cercle de solidarité

14 Lucas Rem ne consacre jamais directement un chapitre ou même un long passage à ses frères et sœur. Néanmoins il les évoque à plusieurs reprises dans la partie la plus personnelle de ses notes consacrée à sa vie, sa formation et ses voyages, que ce soit dans des moments forts de son existence, ou dans des moments beaucoup plus anodins. Sans le dire explicitement et sans jamais utiliser de terme affectueux, le marchand révèle alors l'affection qu'il porte à ses frères et à sa sœur et la force des sentiments qui unissent la fratrie ${ }^{30}$.

En 1519 par exemple, la peste sévit à Augsbourg et Lucas Rem lui-même est très malade et affaibli. Retenu à Augsbourg, vraisemblablement pour ses affaires, il n'en part que le 3 septembre pour rejoindre sa femme, sa mère et ses frères réfugiés tous ensemble à Ulm pour fuir l'épidémie ${ }^{31}$. Quelques années plus tard, en février 1526, Lucas Rem est atteint par la peste et pense mourir ; c'est alors à son frère Endris qu'il recommande très vivement (auf höchst) sa femme, ses enfants et ses biens ${ }^{32}$. Aucun terme affectueux n'est là encore utilisé : pourtant l'amour et la confiance que se portent les frères sont patents. De même ces séjours chez Gilg ou Magdalena au cours de ses voyages d'affaire lui semblent assez marquants pour être notés. On apprend par exemple qu'en 1509 au cours d'un voyage en Italie, Lucas Rem passe sept jours, du 4 au 11 avril, chez son frère 
Gilg qui vit alors à Padoue. Le 2 septembre 1540, c'est chez sa sœur, à Dillingen, qu'il se rend alors qu'il est en déplacement en Allemagne pour ses affaires.

Ces sentiments, toutefois, ne sont pas aussi intenses entre tous les frères et sœur. L'attachement que Lucas Rem porte à Andréas et à Hans avec lesquels il est associé semble plus fort que pour Gilg ou sa sœur. Plus de $90 \%$ des occurrences relatives à la fratrie concernent les deux premiers. Chez les Rem, les sentiments fraternels apparaissent d'autant plus forts que les frères achètent, héritent en commun de biens ou font ensemble des affaires. Les intérêts matériels et le commerce renforcent considérablement le lien fraternel.

Cadeaux et héritages au sein de la fratrie

De fait, Lucas Rem évoque le plus souvent ses frères lorsqu'il aborde des questions matérielles et financières dont il convient manifestement de garder mémoire.

Dans la partie de ses notes intitulée "Mémoire de mes biens ", le marchand précise par exemple qu'en 1502, sa mère lui a donné « en toute liberté et pour son propre usage » 2000 florins, comme à chacun de ses frères; en 1511, à nouveau 1000 florins à chacun des quatre; puis en 1518, encore 500 florins à "chacun de nous, les quatre frères". Dans sa partie intitulée "Les biens que j'ai hérités, les biens que j'ai acquis", le marchand précise également que les frères héritent de certains membres de leur parenté et s'arrangent ensemble pour tirer au mieux profit de leurs biens ${ }^{33}$. Enfin dans sa partie intitulée « Mon contrat de mariage. Ce que j'ai offert. Ce que j'ai reçu. Ce que ma femme m'a apporté », il fait la liste des cadeaux qu'il a faits à ses frères. Pour Endris et sa famille par exemple, il offre à celui-ci et à son fils des morceaux de tissu précieux, à sa femme un bijou d'argent avec leurs armes respectives et enfin, à chacune de ses quatre filles, une robe.

À la mort de Hans en 1527, Lucas et Endris héritent même des biens de leur frère situés à Kissingen. Cet héritage se réalise dans des conditions particulières qui amènent Lucas Rem à en noter tous les détails. Quelques temps avant de mourir, leur frère Hans a en effet acheté un bien foncier et a connu à la suite de cet achat de gros problèmes judiciaires contre la personne du duc Wilhelm de Bavière ${ }^{34}$. Les frais de cette action en justice ont été si importants qu'à sa mort, sa veuve et ses enfants se sont trouvés dans une situation financière extrêmement délicate. "Par amitié (fraintlich), fraternité (bruderlich) et souci de se comporter de manière irréprochable (unverweislich ze handeln) à l'égard de la veuve de notre frère ", Lucas et Hans proposent alors un arrangement à la veuve : lui verser une rente annuelle de vingt florins d'or et récupérer en échange les biens de Hans situés à Kissingen. L'arrangement est conclu le 13 avril 1527 mais Lucas Rem précise que, par respect pour la veuve et ses enfants, les deux frères décident de ne récupérer leurs biens qu'à la fin du mois d'août. Même si la solidarité fraternelle et l'affection portée à la veuve et aux enfants de leur frère ne peuvent pas être totalement déniés, cet arrangement relève aussi d'intérêts bien compris, notamment de la part de Lucas. Dès le mois d'octobre, celui-ci rachète en effet les terres d'Endris pour quatre cents florins et se retrouve ainsi à la tête d'une belle fortune foncière $e^{35}$.

"Lucas Rem et Associés»

Mais ce sont surtout les affaires et la société de commerce que Lucas Rem fonde avec ses frères qui font de la fratrie un espace essentiel dans la vie du marchand. Les relations professionnelles qui unissent Lucas, Endris et Hans renforcent en effet considérablement leur lien fraternel. 
21 De fait, la force de ce lien est antérieure à leur collaboration. Tous trois se forment en effet dans la compagnie des Welser et y débutent leur carrière de marchand. Cette expérience les amène à parcourir l'Europe et à vivre plusieurs mois à l'étranger, dans la péninsule ibérique notamment. Plus tard, devenus maîtres de leur propre société, les trois frères décident d'ailleurs d'investir en Espagne et au Portugal. Leur expérience commune dans ces deux pays y a sans doute beaucoup contribué. Cette période passée au sein de la compagnie des Welser correspond aussi pour les trois frères à des temps difficiles. Les jeunes hommes sont soumis aux décisions parfois arbitraires de leurs supérieurs. Ainsi Lucas Rem raconte que les Welser décidaient selon leur bon vouloir de les envoyer, lui ou ses frères, en Espagne ou en Italie et que, malgré ses demandes, ils ne leur octroyaient jamais de vacances, ne cessant au contraire de les envoyer en mission dans toute l'Europe. Lucas Rem raconte s'être plaint de cette situation pour lui et ses frères auprès de la compagnie à maintes reprises, mais celle-ci lui aurait répondu en des termes « injurieux $»^{36}$.

Par ailleurs, à cette époque, les frères s'échangent de nombreuses lettres dans lesquelles ils s'informent de leurs affaires réciproques. À l'automne 1518 son frère Endris lui écrit pour lui faire part des importantes pertes qu'il subit au Portugal. C'est dans ce contexte que Lucas Rem évoque pour la première fois son souhait de s'associer à ses frères. Mais cette collaboration n'est envisageable que si la société Welser accepte de libérer les trois frères de leurs engagements. Lucas Rem note avoir adressé de nombreuses demandes à la compagnie en ce sens et avoir fait preuve d'une grande persévérance. C'est finalement le 24 décembre 1517 qu'Antonio Welser, patron de la puissante compagnie, consent à délivrer Endris et Lucas de leurs obligations à l'égard de sa société. Hans n'est libéré que quelques mois plus tard, le 9 avril 1518.

Dès lors les trois frères Rem commencent à collaborer au sein de la compagnie qu'ils fondent ensemble ${ }^{37}$. «Le 23 août 1518, au nom de la Sainte et indivise Trinité, moi, mes frères Andréas et Hans et Udrich Handt avons enfin créé notre société. Nous avons passé contrat ensemble et nous sommes engagés pour huit ans, à compter du $1^{\mathrm{er}}$ septembre, à être unis et liés dans cette société ${ }^{38}$. » Comme le note Lucas en 1528, les frères investissent des milliers de florins «ensemble pour les gains comme pour les pertes, ainsi que pour tous les frais. Que Dieu soit avec nous." L'importance des questions financières dans les relations entre les frères explique qu'une grande partie des notes personnelles de Lucas Rem concerne à partir de cette période les investissements, pertes et profits des frères associés. La partie intitulée «mémoire de mes biens » contient essentiellement les bilans annuels de leur société. Il y mentionne les investissements, dépenses, bénéfices et les intérêts faits par chacun des frères dans l'année. Le $1^{\text {er }}$ août 1535 Lucas Rem dit avoir fait ses comptes généraux avec son frère Endris pour l'année 1534; il y note leurs gains et leurs pertes réciproques et précise qu'ils ont réalisé de grands bénéfices en dépit d'importants problèmes, grâce à "leurs efforts, leur travail acharné et leur zèle $»^{39}$.

Liens fraternels et liens professionnels

Cette association dans la sphère professionnelle ne va pas sans poser problèmes quant à la nature de leurs relations. Devenus associés, les frères demeurent-ils avant tout des frères? Leurs relations familiales et l'ordre dans la fratrie influent-t-elle sur leurs relations professionnelles et les hiérarchies internes à la société ? Et si oui, l'aîné a-il des prérogatives et une autorité particulières sur ses cadets? L'aîné, en héritier traditionnel des prérogatives paternelles dans la sphère familiale, peut-il même 
revendiquer «naturellement » le statut de chef de la société comme celui de chef de famille? Dès lors, d'associés, les frères passeraient au statut de frère cadet, puis quasiment de fils; à l'égalité première de l'association se substitueraient des hiérarchies internes héritées de la sphère familiale.

À la lecture des notes de Lucas Rem, il est très net que les relations fraternelles ainsi que l'ordre dans la fratrie jouent beaucoup dans la sphère professionnelle ${ }^{40}$. Aîné, Lucas Rem agit à plusieurs reprises dans le sens des intérêts de ses frères. Il décrit par exemple la façon dont, le 5 février 1510, il s'efforce d'encourager les affaires de son frère Hans lors d'un séjour au Portugal où son frère est représentant de la compagnie des Welser. Au cours de ce séjour, le marchand a le privilège de rencontrer le roi, la reine et leur six enfants et «au moment de prendre congé, il leur baise la main leur recommandant son frère Hans "; la fraternité et les intérêts marchands pouvaient ainsi faire bonne affaire. A plusieurs reprises le marchand prend également la plume au nom de la fratrie pour défendre les intérêts des trois frères dans la compagnie des Welser. En juin 1511, par exemple, le marchand dépense beaucoup d'énergie pour tenter de convaincre la compagnie des Welser de laisser partir son frère de Lisbonne. Malgré cela, au mois d'août, la compagnie décide d'envoyer son frère à Palma. Lucas Rem est furieux et un échange de lettres " très vives et inamicales » s'ensuit entre le marchand et la compagnie, lettres dont Lucas Rem garde des copies. Se révèle ici la dépendance des cadets à l'égard de leur aîné, vraisemblablement plus expérimenté et jouissant d'une plus grande autorité dans la compagnie.

Toutefois, les frères devenus associés, Lucas, l'aîné, se présente assez vite comme le chef "naturel ». Même si cela n'est jamais fait explicitement, tout son discours converge en effet vers l'idée que ses frères lui doivent respect et obéissance ${ }^{41}$. Et le marchand de s'en justifier. Les onze années passées chez les Welser lui ont donné une expérience que ses frères sont loin de posséder. Il s'est efforcé durant toutes ces années d'étudier de manière approfondie le commerce, et de se familiariser avec les techniques marchandes et la clientèle, même si tout cela lui a demandé beaucoup d'efforts. Ainsi lorsqu'il justifie sa demande que la société prenne le nom de Lucas Rem et Associés, c'est à la fois pour rendre hommage à leur père et pour valider sa plus grande expérience $d u$ négoce ${ }^{42}$. De la même manière «l'indocilité » de ses frères, «leur faible expérience » et « leur manque de connaissance dans le domaine des affaires » l'amènent à justifier sa décision unilatérale d'accepter dans leur société un nouveau membre, extérieur à la famille : Jerg Meyting ${ }^{43}$.

À le lire, les statuts de chef de famille et de chef de société se confondent. Son discours sur lui-même dans la compagnie véhicule à maints égards les idéaux d'un bon père, comme le dévouement et l'exigeante bienveillance. Le marchand affirme "n'avoir jamais pensé ni recherché son propre profit ou sa propre renommée mais ceux de ses frères ». Et le marchand de rappeler également le soutien qu'il a volontairement apporté à ses frères afin qu'ils acquièrent expérience et savoir-faire dans le domaine du commerce, en particulier pour les registres comptables. Lucas Rem affirme n'avoir eu alors d'autres soucis que les intérêts de la fratrie et l'honneur du nom des Rem. D'où les reproches faits à ceux-ci qui ne lui manifestent « ni gratitude, ni reconnaissance ».

En même temps qu'il se présente comme un bon père, il décrit à plusieurs reprises ses frères comme de vrais enfants, désobéissants et irresponsables. Quelques mois après la création de leur firme en 1518, Lucas Rem dit de ses frères « qu'ils sont grossiers et lui manquent totalement de respect et de gratitude", à tel point que leur société ne 
connaît plus «ni ordre ni règle $»^{44}$. Excédé par l'attitude de ses frères, il songe même à quitter leur société et à mettre fin au contrat qui les unit. Il lui semble normal d'attendre de ses frères obéissance, respect et reconnaissance. Or ce sont précisément les idéaux de l'époque relatifs aux fils par rapport à leur père. C'est d'ailleurs exactement ce que Lucas Rem lui-même attend de ses propres fils. Pour preuve, son fils naturel Jacob est selon ses termes un "mauvais garçon», " entêté ", " avec un mauvais caractère ». Il se comporte si mal là où son père l'envoie se former et se révèle si " désobéissant » vis-à-vis de ses supérieurs et de son propre père, que Lucas Rem décide finalement de «faire une croix sur lui, en le recommandant à Dieu $»^{45}$. Le discours tenu sur ses frères associés et notamment les termes utilisés sont étonnement comparables.

Pourtant, en réalité, les choses sont plus complexes car les frères ne semblent pas si aisément se soumettre à ce schéma familial et revendiquent manifestement dans la sphère professionnelle autonomie et pouvoir. À travers les récriminations et des reproches de l'aîné, on découvre en effet que les cadets ne suivent pas toujours les projets tout tracés de leur frère. Ainsi en mai 1514 le marchand déplore avec amertume que son frère Hans se soit «facilement laissé convaincre » par la compagnie des Welser de continuer à travailler pour eux et se soit comporté, selon ses notes, de manière « incorrecte » à son égard ${ }^{46}$. Hans ne l'aurait pas prévenu de son projet de s'engager à nouveau auprès des Welser et Lucas note en avoir été très contrarié. Cette décision qu'il présente comme une trahison de la part de son frère, l'aurait même amené à songer à abandonner les affaires ${ }^{47}$. Perce ici la rancœur voire la colère de Lucas Rem face à l'autonomie dont fait preuve son frère. Et l'on peut finalement se demander si ce que Lucas Rem présente à de nombreuses reprises comme de la grossièreté, de la désobéissance et de l'ingratitude de la part de ses frères ne relève pas de leur volonté de nettement différencier espace professionnel et espace familial, relations sociales et relations fraternelles.

30 Finalement, ces notes d'un marchand d'Augsbourg de la fin du Xve siècle et du début du $\mathrm{XVI}^{\mathrm{e}}$ siècle montrent la place fondamentale que les relations fraternelles pouvaient avoir dans le monde marchand à cette époque, notamment en raison des garanties financières et humaines qu'elles offraient. Il convenait de se demander si les liens fraternels et notamment les hiérarchies internes à la fratrie en venaient à structurer la société de commerce. À l'analyse de ce texte, les choses semblent plus subtiles. Le discours de Lucas Rem est celui d'un aîné cherchant à véhiculer l'idée que c'est là l'idéal qui devrait être atteint. Dans ses notes, la société de commerce apparaît comme un prolongement de la famille étroite, légitimement dirigée par le fils aîné devenu chef de famille. Dans ce schéma où famille et société se confondent, les frères cadets associés doivent naturellement manifester obéissance et reconnaissance à leur frère aîné, devenu en quelque sorte leur père et leur patron tout à la fois. Cependant l'analyse critique du discours du marchand permet de se rendre compte que dans le cas de la fratrie des Rem la confusion entre sphère familiale et sphère professionnelle n'est pas si évidente. Les frères cadets font preuve d'indépendance à l'égard de leur aîné et semblent affirmer leurs différences de points de vue, même si Lucas Rem n'évoque jamais ces attitudes qu'en terme d'«irrespect", de "grossièreté " et $\mathrm{d}^{\prime}$ «insoumission». Même si la fraternité est au cœur de l'organisation du monde marchand, l'individualisme et l'esprit d'indépendance des négociants mettent ainsi une limite à la diffusion des hiérarchies familiales traditionnelles hors de la sphère privée de la famille. 


\section{NOTES}

1..D. ALEXANDRE-BIDON et D. LETT, Les Enfants au Moyen Âge Ve-XVe siècles, Paris, 1997 ; D. LETT, « Comment parler à ses filles ? », Médiévales 19, 1990, p. 77-82 ; ID., dir., Etre père au Moyen-Âge, Cahiers de Recherches Médiévales 4, 1997 ; ID., « Tendres souverains », dans Histoire des pères et de la paternité, Paris, 2000, p. 17-39. 2.J. TRICARD, «Les livres de raison français au miroir des livres de famille italiens ; pour relancer une enquête », Revue historique 307, 2002, p. 993-1011.

3..Ch. KLAPISCH-ZUBER, « L'invention du passé familial à Florence (XIV $-\mathrm{XV}^{\mathrm{e}}$ siècles) », dans Temps, mémoire et tradition au Moyen Age, Aix en Provence, 1983 ; EAD., La Maison et le Nom : stratégies et rituels dans l'Italie de la Renaissance, Paris, 1990 ; EAD., « Comptes et mémoire : l'écriture des livres de famille florentins », dans L'Écrit dans la société médiévale. Textes en hommage à Lucie Fossier, Paris, 1991 ; C. CAZALÉ BÉRARD, Ch. KLAPISCHZUBER, « Mémoire de soi et des autres dans les livres de familles italiens », dans R. MORDENTI, C. CAZALÉ BÉRARD, Ch. KLAPISCH-ZUBER, R. BLACK, S. TEUSCHER, « Les livres de famille », Annales Histoire Sciences sociales, juillet-août 2004, p. 805-826.

4..Sources allemandes éditées (liste non exhaustive) : F. FRENSDORFF et M. LEXER, « Chronik des Burkard Zink 1368-1468 », dans Chroniken der deutschen Städte, t. 5, Leipzig, 1866, réed.1965 ; B. GREIFF, « Tagebuch des Lucas Rem aus den Jahren 1494-1541. Ein Beitrag zur Handelsgeschichte des Stadt Augsburg », dans 26. Jahresbericht des Historischen Kreisvereins für Schwaben und Neuburg, Augsbourg, 1861, p. 1-110 ; C. HEGEL, « Ulman Stromers 'Püchel von meim geslechet und von abentewr' », dans Chroniken der deutschen Städte, 1, Leipzig, 1862, rééd. Stuttgart, 1961. Reproduction partielle du manuscrit par L. Kurras, Puechel von mein geslecht und von abentewr : zur 600-Jahrfeier der Gründung der ersten Papiermühle Deutschlands, Stuttgart, 1990 ; Th. VON KERN, « Endres Tucher's Memorialbuch (1421 bis 1440) », dans Chroniken der deutschen Städte, tome 2, Leipzig, 1864, p. 1-53 ;Th. VON KERN, Tucherches Memorialbuch (1386 bis 1451), dans Chroniken der deutschen Städte, tome 10, Leipzig, 1872, p. 1-43 ; W. LOOSE, Anton Tuchers Haushaltbuch (1507 bis 1517), Tübingen, 1877 ; Fr. PFEIFFER, Des schwäbischen Ritters Georg von Ehingen Reisen nach der Ritterschaft, Stuttgart, 1842. Nouvelle édition et commentaire par G. EHRMANN, Göppingen, 1979 ; H. RUPPRICH, Dürer. Schriftlicher Nachlass, Berlin, 1956, p. 7-38 ; GE. STEITZ, « Die Familienchronik Bernhard Rohrbachs aus dem 15. Jahrhundert », AFGK, (II, 2), 1865, p. 404-437 ; GE. STEITZ, « Job Rohrbach's Frankfurter Chronik vom Jahre 1495-1502 », dans AFGK, 1839, p. 1-211; E. ULLMANN, Albrecht Dürer. Schriften und Briefe, Leipzig, 1978, 1989.

5..Voir sur ce point la synthèse de Pierre Monnet sur les débats historiographiques actuels concernant les témoignages autobiographique : P. MONNET, «Petites et grandes gens : un discours des origines dans les témoignages autobiographiques allemands de la fin du Moyen Age ", dans P. BOGLIONI, R. DELORT, C. GAUVARD, Le Petit Peuple dans l'Occident médiéval, Paris, 2002, p. 468-469.

6..Kl. ARNOLD, S. SCHMOLINSKY, U.-M. ZAHND dir., Das dargestellte Ich. Studien zu Selbstzeugnissen des späteren Mittelalters und der frühen Neuzeit, Winkler, 1999 ; Ch. DUHAMELLE, « Les banques de données de témoignages de soi (Selbstzeugnisse), dans l'espace germanique », Bulletin d'Information de la Mission Historique Française en Allemagne, 37, 2001, p. 111-116 ; W. SCHULZE dir., Ego-Dokumente. Annäherung an den Menschen in der Geschichte, Berlin, 1996 ; S. TEUSCHER, « Parenté, politique, comptabilité. Chroniques familiales autour de 1500 (Suisse et Allemagne du Sud) », dans R. MORDENTI, C. CAZALÉ BÉRARD, C. KLAPISCH-ZUBER, R. BLACK, S. TEUSCHER, «Les livres de famille », Annales Histoire Sciences sociales, juillet-août 2004, p. 858. À noter également l'ouvrage fondateur d'H. WENZEL, Die Autobiographie des späten Mittelalters und der frühen Neuzeit, München,1980.

7..M. BEER, « Das Verhältnis zwischen Eltern und ihren jugendlichen Kindern in Spätmittelalterlichen Nürnberg », MVGN, 77, 1990, p. 91-153 ; M. BEER, Eltern und Kinder des späten Mittelalters in ihren Briefen. Familienleben in der Stadt des Spätmittelalters und der frühen Neuzeit mit besonderer Berücksichtigung Nürnbergs, 
Nürnberg, 1990 ; M. BEER, « “Wenn ych eynen naren hett zu eynem mann, da fragen dye freund nyt vyl danach". Private Briefe als Quelle für die Eheschliessung bei den Stadtbürgerlichen Familien des 15. und 16. Jahrhunderts ", dans H.-J. BACHORSKI, Ordnung und Lust. Bilder von Liebe, Ehe und Sexualität in Spätmittelalter und früher Neuzeit, Trêves, 1991 ; E. MASCHKE, « Die Familie in der deutschen Stadt des späten Mittelalters », dans Sitzungsberichte des Heidelberger Akademie, 1980, p. 9-98 ; P. MONNET, « La ville et le nom : le livre des Melem, une source pour l'histoire privée des élites francfortoises à la fin du Moyen Age », Le Journal des Savants, juin/décembre 1999, p. 491-539 ; G. ROHMANN, Eines Ehrbaren Rathts gehorsamer amptmann. Clemens Jäger und die Geschichtsschreibung des 16. Jahrhunderts, Augsbourg, 2001 ; U.-M. ZAHND, Die autobiographischen Aufzeichnungen des Ludwig von Diesbachs. Studien zur spätmittelalterlichen Selbstdarstellung im Oberdeutschen und schweizerischen Raume, Berne, 1986 ; U.-M. ZAHND « Einige Bemerkungen zu spätmittelalterlichen Familienbüchern aus Nürnberg und Bern », dans Nürnberg und Berne. Zwei Reichsstädte und ihre Landgebiete, Erlangen, 1990 ; U.-M. ZAHND, « Stadtchroniken und autobiographische Mitteilungen. Studien zur Selbstdarstellung spätmittelalterlicher Bürger », dans Kl. ARNOLD, S. SCHMOLINSKY, U.-M. ZAHND, Das dargestellte Ich. U.-M. ZAHND, « Ehegatten in spätmittelalterlichen Selbstzeugnissen », dans Ch. HESSE, B. IMMENHAUSER, O. LANDOLT, B. STUDER, Personen der Geschichte, Geschichte der Personen, Berne, 2003, p. 201-220.

8..C. HEGEL, « Ulman Stromers 'Püchel von meim geslechet und von abentewr' », loc. cit.

9..G.E. STEITZ, « Die Familienchronik Bernhard Rohrbachs... », loc. cit.

10..Th. von KERN, Endres Tucher's Memorialbuch, op.cit.

11.B. GREIFF, Tagebuch des Lucas Rem..., op. cit.

12..F. FRENSDORFF et M. LEXER, Chronik des Burkard Zink..., loc. cit.

13..Précisons toutefois que les deux hommes n'ont pas le même niveau de richesse, Lucas Rem ayant un niveau de fortune bien plus élevé que Burkard Zink.

14..Leur profil est de ce fait comparable aux auteurs des ricordanze italiennes étudiées par Christiane Klapisch-Zuber. « Les ricordanze sont l'œuvre et parlent surtout de privilégiés, hommes des villes bien nourris et éduqués, qui ont de quoi élever leurs enfants, mènent des affaires où ils investissent et calculent constamment, occupent des fonctions dans l'arène politique ", Ch. KLAPISCH-ZUBER, La Maison et le Nom, op., cit.p. 9.

15..H. RUPPRICH, Dürer. Schriftlicher Nachlass, op. cit. ; E. ULLMANN, Albrecht Dürer. Schriften und Briefe, op. cit. ; P. VAISSE, Dürer. Lettres, écrits théoriques et traité des proportions, Paris, 1964.

16..O. G. OEXLE, « Memoria als Kultur », dans Memoria als Kultur, Göttingen, 1995 ; P. MONNET, « Elites dirigeantes et distinction sociale à Francfort-sur-le-Main », Francia 27, 2000, p. 117-162.

17..S. TEUSCHER, « Parenté, politique, comptabilité. Chroniques familiales autour de 1500 (Suisse et Allemagne du Sud) », dans R. MORDENTI, Cl. CAZALÉ BÉRARD, C. KLAPISCH-ZUBER, R. BLACK, S. TEUSCHER, « Les livres de famille », Annales Histoire Sciences sociales, juillet-août 2004, p. 858.

18..P. MONNET, « Elites dirigeantes et distinction sociale à Francfort-sur-le-Main », loc. cit. ;P. Monnet, « La mémoire des élites urbaines dans l'Empire à la fin du Moyen Âge entre écriture de soi et histoire de la cité », dans H. BRAND, P. MONNET, M. STAUB, J. THORBECKE dir., Memoria, Communitas, Civitas. Mémoire et conscience urbaines en Occident à la fin du Moyen Age, 2003, p. 49-72 ; P. MONNET, « La monographie familiale entre histoire urbaine et histoire culturelle : l'exemple des pays germanies de l'Empire à la fin du Moyen Âge », dans M. AURELL dir., Le Médiéviste et la Monographie familiale : sources, méthodes, problématiques, Turnhout, 2004, p. 37-52.

19..Ceci est à mettre en lien avec la révolution documentaire qui a lieu à cette époque dans les villes allemandes.

20..P. MONNET, Les Rohrbach de Francfort. Pouvoirs, affaires et parenté à l'aube de la Renaissance allemande, Genève, 1997, p.12. 
21..B. GREIFF, Tagebuch des Lucas Rem, op. cit., p. 1.

22..B. GREIFF, Tagebuch des Lucas Rem, op. cit., p. 2.

23..P. MONNET, « La mémoire des élites urbaines », loc. cit., p. 61.

24..Comme l'écrit Sophie CASSAGNES-BROUQUET au sujet des frères Dombet, peintres avignonnais du XV ${ }^{\mathrm{e}}$ siècle : «Quand deux frères ont opté pour la même spécialité artistique que leur père, la fratrie prend alors une tout autre dimension, elle peut désormais se révéler comme un mode d'association aussi bien familial que professionnel. » S. CASSAGNES-BROUQUET, « Les liens familiaux au cœur du fonctionnement des milieux artistiques en Europe du Nord-Ouest à la fin du Moyen Age ", dans M. AURELL dir., Le Médiéviste et la Monographie familiale, op. cit., 2004, p. 215.

25..Voir l'étude d'Elmar Lutz sur les contrats de société. E. LUTZ, Die rechtliche Struktur süddeutscher Handelsgesellschaften in der Zeit der Fugger, Tübingen, 1976, 2 vol.

26..L'appellation de « journal » donné par celui qui l'a publié au XIX ${ }^{\mathrm{e}}$ siècle, B. Greiff, n'est pas entièrement satisfaisante. Bien que le livre de Lucas Rem semble avoir été constitué jour après jour et que celui-ci consigne la date précise de chaque événement, la forte différenciation thématique qu'on observe dans ces notes correspond davantage à la forme des ricordanze toscanes.

27..B. GREIFF, Tagebuch des Lucas Rem, op. cit., 1861.

28..D. LETT, « Brothers and sisters. New perspectives on Medieval Family History », dans K. MUSTAKALLIO, J. HANSKA, H.-L. SAINIO et V. VUOLANTO dir., Hoping for Continuity. Childhood, Education and Death in Antiquity and the Middle Ages, Acta Instituti Romani Finlandiae-33, Rome, 2005, p. 19.

29..A.-M. CERTIN, Paternité et société en Allemagne à la fin du Moyen Âge, Mémoire de DEA sous la direction de JC. Schmitt, Paris, EHESS, 2005, p. 98.

30..D. LETT, « Brothers and sisters. New perspectives on Medieval Family History », op. cit., p. 21.

31..B. GREIFF, Tagebuch des Lucas Rem, op. cit., p. 23

32..Ibid, p. 26

33..Ibid, p. 57 et 62 .

34..Ibid, p. 60

35..Ibid, p. 60

36..Ibid, p. 20

37..Notons chez les Rem le caractère pérenne de cette association de frères, ce qui n'est pas le cas par exemple de l'association entre les frères Dombet, peintres d'Avignon au XV siècle, étudiée par Sophie Cassagnes-Brouquet. L'alliance apparaît dans ce cas comme une assurance temporaire pour des hommes encore jeunes. Lorsqu'ils se sentent assez sûrs d'eux, les deux frères Albéric et Jacques rompent leur association. $C f$. S. CASSAGNES-BROUQUET, « Les liens familiaux au cœur du fonctionnement des milieux artistiques... ", loc. cit., p. 216

38..B. GREIFF, Tagebuch des Lucas Rem, op. cit., p. 31.

39..Ibid, p. 39.

40..Sur l'influence des écarts d'âge dans la fratrie, voir D. LETT, « Vieux frères et oncles jeunes : écart de générations et écarts d'âge dans les familles de la fin du Moyen Âge », dans J.-P. BARDET, J.-L. LUC, I. ROBINROMERO et C. ROLLET dir., Lorsque l'enfant grandit. Entre dépendance et autonomie, Paris, 2003, p. 93-103.

41..Les caractéristiques de cette relation se retrouvent sur de nombreux points dans celle tissée par Jean Gerson, chancelier de l'Université de Paris du XV ${ }^{\mathrm{e}}$ siècle, avec ses frères et sœurs, F. LEDWIGE, « Relations de famille dans la correspondance de Gerson », Revue historique, 271, Paris, 1984, p. 13-18.

42..B. GREIFF, Tagebuch des Lucas Rem, op. cit., note 226, p. 104.

43..Ibid, p. 31 .

44..Ibid, p. 32 .

45..Ibid, p. 65 
46..Ibid, p. 17

47..Ibid, p. 18

\section{RÉSUMÉS}

Dans l'espace germanique, les écrits privés (autobiographie, journaux ou livres de famille) se comptent en grand nombre. Si ces sources sont l'objet d'un fort regain d'intérêt en Allemagne depuis les années 1990, notamment dans le domaine de l'histoire de la famille, elles restent en revanche assez méconnues en France. L'étude des notes personnelles de Lucas Rem (1481-1542), marchand d'Augsbourg, permet d'étudier le poids de la fratrie dans la sphère du commerce. La société de commerce apparaît comme un prolongement de la famille étroite, légitimement dirigée par le fils aîné, promu chef de famille. Les frères cadets associés doivent manifester obéissance et respect à leur frère aîné, devenu en quelque sorte leur père et leur patron tout à la fois. Cependant l'analyse critique du discours du marchand permet d'observer que les frères cadets n'acceptent pas totalement cette confusion entre sphère familiale et sphère professionnelle.

Professional and fraternal relations according to Lucas Rem's journal, merchant of Augsburg (1481-1542). In Germany, private documents (autobiographies, journals, family books...) are numerous. Whereas they have been often studied in Germany since 1990, specially in the field of family history, they globally remain unknown in France. By studying the personal notes of Lucas Rem (1481-1542), merchant of Augsburg, we will see the importance of sibship in trade. The trading company appears as extension of the nuclear family. And this one seems to be run by the eldest brother, promoted to headman in the family. The youngest brothers have to be obedient and respectful to their eldest brother, who becomes at the same time their father and their manager. Nevertheless the analysis of the words of the merchant shows that the younger brothers do not necessarily agree to this confusion between family and business.

\section{INDEX}

Mots-clés : Allemagne, écrit privé, fratrie, marchand, société de commerce

Keywords : Augsbourg, brothers, Germany, merchant, private documents, trading company

\section{AUTEUR}

AUDE-MARIE CERTIN

EHESS/GAHOM, 2 rue Vivienne, 75002 Paris 